\title{
A comparison of recollective memory and source monitoring
}

\author{
WAYNE DONALDSON, THERESA M. MACKENZIE, and CATHY F. UNDERHILL \\ University of New Brunswick, Fredericton, New Brunswick, Canada
}

\begin{abstract}
Participants in recognition memory experiments are often asked for additional information about their memories after each positive recognition response. Two experiments were conducted that were identical, except that the first asked participants to make remember/familiar judgments about the items they recognized, and the second asked them to make source judgments. The similarity between the recollection and source judgment data has implications for measurement issues and theory development.
\end{abstract}

The standard yes/no recognition memory task presents participants with items, such as words, and asks them to decide whether each item occurred previously in the experiment. After saying "yes" or "no" to an item, and perhaps providing a confidence rating, the person goes on to the next item. In real life, however, a recognition response is often not sufficient. An eyewitness must go beyond recognition and remember that the face belonged to the appropriate person at the crime scene. A person in therapy must discriminate between memories of imagined and real events. A reporter must remember who told him/her the story.

Two developing areas of research attempt to go beyond the recognition response to gain more information about the memory. In one case, participants are asked to follow a recognition response with an indication of whether they actually remember some details about the prior occurrence of the item or whether they just know it was there (e.g., Gardiner \& Java, 1993; Tulving, 1985). In the other case, participants are asked to follow a recognition response with an indication of the source of the memory (e.g., Johnson, Hashtroudi, \& Lindsay, 1993). Both techniques require participants to make a postrecognition judgment about the nature of the memory that allowed them to recognize the item. Remember/know judgments require recollection of any details of the prior occurrence. Source judgments require a specific recollection about the source of the prior occurrence.

The two types of judgments should be related. A person who says "I remember" may be basing that response on something other than source information, but a person who remembers the source should be able to say "I remember." In fact, Johnson et al. (1993) suggest that know or familiarity responses occur when a person remembers virtually no information about the source.

The authors would like to thank Larry L. Jacoby, John T. Wixted, Marcia K. Johnson, and Henry L. Roediger III for their thorough reviews and insightful comments on an earlier version of this paper. Correspondence should be addressed to W. Donaldson, Department of Psychology, University of New Brunswick, Fredericton, NB, Canada E3B 6E4 (e-mail: donaldsn@unb.ca).
In both the recollective memory and source judgment literature, there are ongoing discussions about measurement and theory issues. One theory issue is whether people making remember/know judgments are accessing different memory systems (Donaldson, 1996; Gardiner \& Java, 1993). The appropriate measure of source memory is also a topic of debate (Batchelder \& Riefer, 1990; Kinchla, 1994; Murnane \& Bayen, 1996). The present paper does not directly address those complex issues. Rather, we wish simply to compare performance in the recollective memory and source monitoring tasks to see how similar they might be.

Two experiments were conducted using the Jacoby (1983) manipulation of encoding conditions expanded upon in Blaxton (1989). The two experiments were identical except for the postrecognition response required of the participants. In Experiment 1, participants made recollection decisions; in Experiment 2, they indicated the encoding condition under which each recognized item had previously been studied.

\section{EXPERIMENT 1}

\section{Method}

Participants and Materials. The participants were 42 undergraduates recruited from introductory psychology courses. A set of 156 highfrequency words was selected, 12 of which served as buffer items in the presentation and test lists. The remaining 144 words were randomly divided into two sets of 72, each set providing study items for half of the participants and distractors for the other half. Within a study list, words were randomly assigned to one of three encoding conditions so that each study list contained 24 examples of each condition. In the generate condition, the participants saw a descriptive phrase and the target word, which had from one to three letters deleted (e.g., QUANTITY OF EGGS $D_{-}$EN). In the context condition, both were presented (QUANTITY OF EGGS DOZEN). In the read condition, asterisks $(* * * * * * * * * * * * * * *$ DOZEN) replaced the description. The order in which conditions appeared in the presentation list was random. Words were rotated across the different conditions in six different presentation lists so that each word was used in all conditions. The single common test list consisted of all 156 words. The 12 buffer words were presented at the beginning of the test list to verify the participants' understanding of test instructions but were not analyzed.

All items appeared on a computer screen, the descriptive phrase near the top and the to-be-remembered word in the center. The items were each shown for $5 \mathrm{sec}$, with a $0.5-\mathrm{sec}$ delay between items. In the self- 
paced test, each word appeared individually in the center of the screen. Throughout the test the numbers $0,1,2,3$ were spaced across the bottom of the screen. Directly below the numbers were the category labels no, yes unsure, yes sure, and yes very sure, respectively.

Procedure. The participants were tested individually and were randomly assigned to one of the six presentation lists. The three presentation formats were described to the participants, who were instructed to read everything on the screen aloud. They were also told that they would be given a memory test only on the words. All participants received the same test list. Each word was read aloud by the participant and was followed by a recognition response based on the numbers $0,1,2,3$. The scale is the same as that used by Donaldson (1996). It produces liberal responding and provides confidence ratings on "yes" responses, the responses of interest. After each nonzero recognition response, the participants were asked to make a recollective judgment, to indicate if they remembered the word or if it just looked familiar. As in Donaldson (1996), familiar rather than know was used to indicate nonrecollection, because the word know carries a connotation of certainty that is inconsistent with a confidence rating that indicates lack of certainty. Participants find it hard to say that they are unsure that an item was there but that they know it was. The remember response was for recognition accompanied by a conscious recollection of the prior occurrence of the word. The participants were given extensive instructions as to the use of the remember/ familiar distinction (Gardiner, 1988). The recognition test was selfpaced, with the participant simply pressing the enter key to see the next word after he or she had finished responding to the word on the screen.

\section{Results}

The overall recognition results replicate the effects found by Jacoby (1983) and are shown in Table 1. The generate condition produced a higher hit rate $(.97)$ than did the context condition (.87), which in turn was larger than that in the read condition (.72). In addition, the remember and familiar data are very similar to the dissociation Jacoby found between his explicit recognition task and his implicit perceptual identification task. When people said they remembered, hit rates decreased across the generate (.76), context (.58), and read (.31) conditions. When people responded familiar, performance increased across the three conditions, from .21 to .28 to .41 . These results nicely parallel those found by Java (1994) in a paper that was brought to our attention while this one was in final revision. Java (1994, Experiment 2) compared the generate and read conditions. She had subjects follow cued recall with remember/know decisions and found a higher rate of remember responses to generated items (.32) than to read items (.09) and a lower rate of know responses to generate items (.09) than to read items (.13). An interpretation following Gardiner and Java (1993) would suggest that remember responses tap the same resources as explicit memory tasks, whereas familiar responses are indicative of implicit memory.
An alternate interpretation would account for the dissociation between remember and familiar responses in terms of decision processes (Donaldson, 1996). The hypothetical distributions in Figure 1 are like those in signal detection theory and are elaborations of those in Donaldson (1996). The three distributions, from left to right, represent the read, context, and generate conditions, respectively, mapped onto a recognition strength dimension. The new-item distribution has been omitted. The top panel shows the liberal no/yes criterion placement that produces the overall recognition data. The areas under the three distributions and to the right of that criterion represent the three recognition hit rates. The area under the generate distribution is larger than that under context, which is in turn larger than the area under the read distribution, mirroring the actual hit rates. The criterion to the right, the placement of which is also under the control of the participant, divides the recognized responses into those that the participants are willing to say they remember and those they call familiar. Items to the right of the familiar/ remember criterion are given a remember response. Again, the area under the generate distribution is larger than that under context, which is larger than under read, as in the remember data. The areas under the distributions and between the criteria represent the familiar responses. Normally, one would not consider areas between criteria, but, by the model, that is what familiar responses represent. As in the data, when people say "familiar," the area under the read distribution and between the two criteria is larger than that under the context distribution, which is in turn larger than that under the generate distribution. The dissociation between remember and familiar responses can thus be derived from a single strength model. The same model also handles parallel increases in remember and know judgments as a function of number of presentations as reported by Gardiner, Kaminska, Dixon, and Java (1996). The model allows such parallel increases in the remember/know components when overall performance level increases as a function of presentations.

Like the recognition study in Donaldson (1996), this study also included confidence ratings. One can examine the remember/familiar distinction under conservative yes/no responding ( +3 ratings), when the participants said that they were very sure an item was old. These data show the relationship between the three conditions to be unchanged when the participants said they remembered. The high-confident remember hit rates for the generate, context, and read conditions were unchanged from the

Table 1

Hit Rates, False-Alarm (FA) Rates, and $A^{\prime}$ Values for Overall Recognition in Both Experiments and for Recollection and Nonrecollection (Experiment 1) and Correct and Incorrect Source (Experiment 2)

\begin{tabular}{|c|c|c|c|c|c|c|c|c|c|c|c|c|c|c|c|c|c|c|}
\hline \multirow[b]{3}{*}{ Condition } & \multicolumn{9}{|c|}{ Experiment 1} & \multicolumn{9}{|c|}{ Experiment 2} \\
\hline & \multicolumn{3}{|c|}{ Recognition } & \multicolumn{3}{|c|}{ Remember } & \multicolumn{3}{|c|}{ Familiar } & \multicolumn{3}{|c|}{ Recognition } & \multicolumn{3}{|c|}{ Source Correct } & \multicolumn{3}{|c|}{ Source Incorrect } \\
\hline & $\mathrm{Hit}$ & FA & $A^{\prime}$ & Hit & FA & $A^{\prime}$ & $\mathrm{Hit}$ & $\overline{F A}$ & $A^{\prime}$ & Hit & FA & $A^{\prime}$ & Hit & $\overline{F A}$ & $A^{\prime}$ & Hit & FA & $\overline{A^{\prime}}$ \\
\hline $\mathrm{R}$ & 0.72 & 0.16 & 08 & 0 & & & & & & & & 0. & & & 0.84 & 0.09 & 0.04 & 0 \\
\hline Cont & 0 & 0.16 & 0.91 & 0.58 & 0.8 & 0.89 & 0.28 & 0.14 & 0.66 & 0.88 & & 0.91 & 0.61 & 0.03 & 0.89 & 0.26 & 0.15 & \\
\hline Generate & 0.97 & 0.16 & 0.95 & 0.76 & 0.02 & 0.93 & 0.21 & 0.14 & 0.60 & 0.96 & 0.18 & 0.94 & 0.76 & 0.01 & 0.94 & 0.19 & 0.17 & 0.5 \\
\hline
\end{tabular}



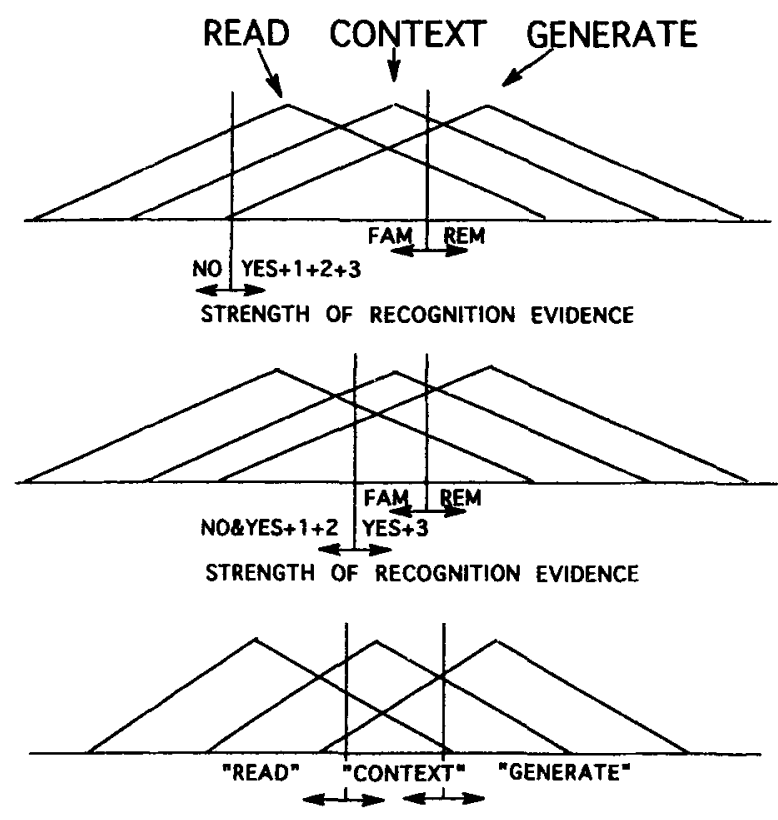

STRENGTH OF SOURCE EVIDENCE

Figure 1. Schematic diagrams showing the effect of response criterion on recognition and postrecognition response rates. The three distributions represent the old items from the read, context, and generate encoding conditions. The top two panels illustrate the recollective task in Experiment 1. The top panel shows the yes/no recognition criterion placed liberally. The middle panel shows the conservative very sure $(+3)$ criterion placement. The bottom panel shows three very similar distributions placed on a "source" dimension rather than a "recognition" dimension. The criteria placed on those distributions determine source identification responses. See text for further details.

overall hit rates at $.75, .57$, and .31 , respectively. However, the reverse depth effect found with familiar responses has disappeared in the high-confident data. The familiar hit rates for the generate, context, and read conditions were $.14, .13$, and .16 , respectively. The middle panel of Figure 1 shows the more conservative very sure $(+3)$ criterion placement. The areas between the two criteria, representing the familiar responses, are smaller and much more similar in size, as occurred in the conservative familiar data.

Thus, while the overall remember/familiar data nicely parallel Jacoby's (1983) explicit/implicit results, a change in the yes/no criterion changes the nature of the dissociation. These data, along with most other recollective memory data sets, are nicely handled by a decision process model (Donaldson, 1996). The model is not meant to and cannot account for the original Jacoby dissociation where the implicit and explicit measures are quite independent of one another. But it can account for the remember/familiar data, which are not independent of each other.

\section{EXPERIMENT 2}

\section{Method}

Experiment 2 ( $n=54$ ) was identical to Experiment 1, except that, following a nonzero recognition response, the participants were asked for a source judgment rather than a remember/familiar response. Prior to the test, each person was reminded of the three types of presentations they had seen (read, context, and generate) and was asked to indicate, for each word recognized, in which condition they thought it had originally been presented.

\section{Results}

The basic data are shown in Table 1. The first thing to note is that overall recognition performance was unaffected by the nature of the postrecognition response. This was so whether one looks just at hit rates or at $A^{\prime}$, an estimate of the area under the isosensitivity curve.

The interesting comparison is between the Experiment 1 data when people said they remembered and the Experiment 2 data when they correctly identified the source of the item. For generated items, the hit rates indicate that the proportion above the remember/familiar criterion was .76 , and the proportion where the source was correctly identified was .76. In the context condition, the comparable proportions were .58 and .61 . But in the read condition, although the proportion that people were willing to say they remembered was only .31 , they correctly identified the source at a rate of .64. How can people be so good at remembering source when they are unwilling to say they remember? It seems clear that at least three different things could account for people identifying items as coming from the read condition. First, some proportion of the time (perhaps as much as .31), people might actually remember that items were in the read condition. Second, read responses might be the result of an inference, where recognized items known to have been in neither the generate nor the context condition must have been in the read condition. Third, the read response is the default response (Rabinowitz, 1989) when the item is recognized but no source information is available. In that sense, it is similar to the familiar response in Experiment 1, which is the default response when an item is above the yes/no criterion but does not exceed the remember/ familiar criterion (i.e., when the participant is unwilling to say that he or she remembers).

Two types of evidence point to the latter similarity between familiar and read responses. First, one can look at false-alarm rates. In Experiment 1, new items incorrectly classified as old were mainly classified as familiar (.14 familiar out of .16 false alarms). In Experiment 2, most false alarms were identified as having been in the read condition (.14 of the .18 false alarms). Second, familiar response rates are very dependent on the yes/no response criterion (Donaldson, 1996, and Experiment 1 above). They drop dramatically when one moves from the overall recognition data to the very sure $(+3)$ data. The proportion of times people identify read items as having been in the read condition is similarly affected. In the overall recognition data, that proportion is .64 but falls to .44 in the high-confident +3 data. Similar declines do not occur when people identify items as having come from the generate or context conditions. The use of the read response was affected by the placement of the yes/no criterion adopted, paralleling the use of the familiarity response. The participants seldom identified the generate 
or context conditions as the source of an item unless they had given a recognition rating of +3 . This parallels Experiment 1 , where remember responses seldom followed other than +3 recognition. Following a recognition rating of +2 or +1 , the source response was almost always read, and the recollective response was usually familiar.

To compare the proportion of read items to which people give a remember response (.31) and the proportion for which the source judgment was correct (.64) is inappropriate because of the vastly different false-alarm rates. In Experiment 1, there was a common false-alarm rate for recollective memory (.02), the proportion of new items called old and identified as being remembered. In Experiment 2, however, the false-alarm rates for source memory were different for the three conditions. For the read condition, the false-alarm rate was the proportion of new items called old and identified as having been in the read condition. Similarly, the false-alarm rates for the context and generate conditions were new items called old and identified as being context and generate items, respectively. Table 1 shows those false-alarm rates and the $A^{\prime}$ values (Donaldson, 1992). The $A^{\prime}$ values for the three conditions when people say they remember are very similar to those when they correctly identify the source. Table 1 also shows $A^{\prime}$ values for the familiar responses in Experiment $l$ and the incorrect source responses in Experiment 2. While the decision model does make predictions about those values (see Donaldson, 1996, for details), it should be clear that they do not represent the area under an isosensitivity curve.

\section{DISCUSSION}

Clearly, the numbers are very similar when people are asked to say whether they remember and when they are asked to identify the source of an item. The only real discrepancy in the hit rates occurs in the read condition. People say "read" when they are reluctant to say "generate" or "context" in much the same way that people say "familiar" when they are reluctant to say "remember." The use of "read" as a default response obviously contaminates measures of source monitoring for that condition. The calculation of $A^{\prime}$ values that take into account different falsealarm rates eliminates the differences.

Given the striking similarity between the two data sets, it might be useful to consider similar models. Donaldson (1996) has suggested that when people are asked to distinguish between what they remember and what just looks familiar, they make that decision using the same'mnemonic information that supported their original recognition response. Clearly, the same cannot be done when people are asked to identify the source of an item. What prevents it from working is the situation in which information from two different sources is not differentially recognizable (i.e., where the old item distributions on the recognition dimension are distinguishable from new items but not from each other). Nevertheless, participants can differentiate the sources at a level above chance. As one example, Light, LaVoie, Valencia-Laver, Owens, and Mead (1992) report data from two experiments using modality of presentation to establish different sources. Recognition performance did not differ for items that were seen and items that were heard, but the ability to identify whether they had been seen or heard, was always well above chance. On a dimension of recognition strength, the visual and auditory items would be indistinguishable but they are nevertheless distinguished.

Since source is only one component of the strength of recognition evidence, participants faced with the need to make a source judgment may consider the items on a more restricted dimension, reflecting only the strength of source evidence. Criteria can then be established on that dimension to guide source decisions. The bottom part of Figure 1 shows such distributions. Where there are recognition differences between items from different sources, as in these experiments, the recognition and source distributions may look similar. Items above the criterion to the right will be identified as having come from the generate condition, those between the criteria will be called context items, and those below both will be identified as read items. One aspect of the present data is clearly in line with such a model. When the participants made a source error on an item from the generate condition, they were more likely to say "context" than to say "read" (.13 vs. .07). Source errors on context items were .11 generate and .16 read. Source errors to items that were originally in the read condition were .07 context and only .01 generate. These numbers are reflected in the distributions in Figure 1. In the present case, criteria could have been placed on the original recognition dimension that would have reflected this pattern of errors. Such could not have been done had there been no recognition differences but still some source memory, thus requiring a strength of source evidence dimension.

So what is the relationship between recognition, recollection, and source judgments? Most researchers seem satisfied with the idea that recognition data cannot be understood without taking decision criteria into account, that participants generate yes and no responses by establishing some criterion on a continuous strength dimension. Even though the language we use echoes a discrete state model (i.e., we say that an item is recognized or not), the underlying model is more often one in which a person says he/she recognizes an item because it exceeds some criterion. To then ask whether such items are remembered-. whether some aspect of the encoding conditions can be recollected-invites the participant to consider a number of possible encoding dimensions and to say "I remember" if some more stringent criterion is exceeded. At least to date, recollective data seem to map onto the original "strength of recognition" dimension (Donaldson, 1996). At some point, data may be found that do not. It would then be necessary to consider either models where recollective decisions are made on a more restricted dimension, as suggested above for the source monitoring task, or models that have both discrete-state and continuous-dimension components (Yonelinas, 1994).

One of the encoding dimensions that can undoubtedly enter into recollective decisions involves information about the source of a memory. Where different sources are not differentially recognizable (Light et al., 1992), one would not expect to find differences in recollection. There could, however, be differentiation of sources. Thus, source monitoring cannot be mapped onto the overall strength of recognition dimension. Making a source judgment can still be viewed as the result of a decision process where criteria are established to guide responding. When different sources produce large differences on the recognition dimension, as in the present study, source will be a major component of the recollective decision, and, thus, there will be a high degree of similarity between recollective judgments and source monitoring data.

\section{REFERENCES}

Batchelder, W. H., \& Riefer, D. M. (1990). Multinomial processing models of source monitoring. Psychological Review, 97, 548-564.

BlAXTON, T. A. (1989). Investigating dissociations among memory measures: Support for a transfer-appropriate processing framework. Journal of Experimental Psychology: Learning, Memory, \& Cognition, 15, 657-668.

DONALDSON, W. (1992). Measuring recognition memory. Journal of Experimental Psychology: General, 121, 275-277.

DONALDSON, W. (1996). The role of decision processes in remembering and knowing. Memory \& Cognition, 24, 523-533.

GaRDINER, J. M. (1988). Functional aspects of recollective experience Memory \& Cognition, 16, 309-313.

GaRDiner, J. M., \& JAVA, R. I. (1993). Recognizing and remembering. In A. F. Collins, S. E. Gathercole, M. A. Conway, \& P. E. Morris (Eds.), Theories of memory (pp. 163-188). Hillsdale, NJ: Erlbaum.

Gardiner, J. M., Kaminska, Z., Dixon, M., \& Java, R. I. (1996). Repetition of previously novel melodies sometimes increases both remember and know responses in recognition memory. Psychonomic Bulletin \& Review, 3, 366-371.

JACOBY, L. L. (1983). Remembering the data: Analyzing interactive pro- 
cesses in reading. Journal of Verbal Learning \& Verbal Behavior, 22, 485-508.

JAVA, R. I. (1994). States of awareness following word stem completion European Journal of Cognitive Psychology, 6, 77-92.

JohnSON, M. K., HaShTroudi, S., \& LindSaY, D. S. (1993). Source monitoring. Psychological Review, 114, 3-28.

Kinchla, R. A. (1994). Comments on Batchelder and Riefer's multinomial model for source monitoring. Psychological Review, 101, 166-171.

Light, L. L., La Voie, D., Valencia-Laver, D., Owens, S. A. A., \& MEAD, G. (1992). Direct and indirect measures of memory for modality in young and older adults. Journal of Experimental Psychology: Learning, Memory, \& Cognition, 18, 1284-1297.
Murnane, K., \& Bayen, U. J. (1996). An evaluation of empirical measures of source identification. Memory \& Cognition, 24, 417-428.

RABINOWITZ, J. C. (1989). Judgments of origin and generation effects: Comparisons between young and elderly adults. Psychology \& Aging, 4, 259-268.

Tulving, E. (1985). Memory and consciousness. Canadian Psychology, 26, 1-12.

YonelinAS, A. P. (1994). Receiver-operating characteristics in recognition memory: Evidence for a dual-process model. Journal of Experimental Psychology: Learning, Memory, \& Cognition, 20, 1341-1354.

(Manuscript received October 22, 1995; revision accepted for publication April 3, 1996.) 